\title{
A 57 year Old Man with Depressive Disorder, Congestive Heart Failure and Non Adherence with Treatment
}

\author{
Nazmie F Ibishi $^{1 *}$, Nebi R Musliu², Shpend Haxhibeqiri ${ }^{3}$, Bujar Obertinca ${ }^{4}$ \\ ${ }^{1}$ Fama College, Clinic of Psychiatry, Prishtina, Kosova \\ ${ }^{2}$ UCCK, Clinic of Cardiology, Prishtina, Kosova \\ ${ }^{3}$ UCCK, Institute of Kosovo Forensic Psychiatry, Kosovo \\ ${ }^{4}$ Lecturer at Heimerer College, Prishtina, Kosovo
}

"Corresponding Author: Nazmie F Ibishi, Fama College, Clinic of Psychiatry, Prishtina, Kosova, E-mail: nazmiemb3@hotmail.com

Received: 04 December 2018; Accepted: 27 December 2018; Published: 09 January 2019

\begin{abstract}
Behaviors that decline the quality of life and shorten it, are in different forms. Some of the potential behaviors that are life threatening are cigarette smoking, risks during drive and ignoring the doctor recommendations. Suicide behavior that do not have a clear and identified intentionality, can often be indirect behavior leading to suicide. Depression may increase the risk of cardiac crises and death through direct pathophysiological mechanisms of neurohormonal activity, inflammation, cardiac arrhythmia or through the patient's behavioral mechanism. Depressed patients have difficulty in therapeutic compliance and in changing their lifestyle adequately. This case report is an important lesson for health care providers and challenging they face with management strategy including poor adherence with treatment in patients with congestive heart failure (CHF) and potential failing to recognize the early symptoms of depression as independent risk factor for mortality and morbidity in CHF.
\end{abstract}

Keywords: Congestive heart failure; Suicide; Depression

\section{Case Presentation}

A 57 year old man presented in our ambulatory setting with three day history of chest pain accompanied with nocturnal cough and palpitations. His past medical treatment history of HF was remarkable with medication taking 
off. The patient was found, upon further evaluation, to have significant paroxysmal nocturnal dyspnea and neck vein distension.

\section{Investigation}

2.1 Chest radiography: Chest radiography showed increased heart silhouette.

2.2 ECG: ECG shows abnormal changes with intraventricular conduct system disturbance. Heart rate 123 beats/min.

2.3 Echocardiography: Echocardiography detected moderate mitral stenosis with moderate mitral regurgitation. Mild tricuspidal regurgitation. EjectionFraction $<31 \%$. Mild grade of pulmonary hypertension. Left ventricular and atrial cavity was dilated. Left ventricular abnormal kinesis with reduction of systolic function. Right ventricular cavity dilated. Moderate pericardial effusion.

\subsection{Dg. Cardiopathia congestiva idiopathica. Heart Failure NYHA III-IV}

Psychiatric mental examinations was underwent in outpatient setting and shows that, his mood was primarily anxious and depressed both subjectively and objectively. He expressed behavioral agitation, restlessness and feeling of breathlessness with palpitations and sweating. In cognitive function showed difficulties in concentration and poor social interaction as result of lack of interest and violation He expressed poor self esteem. He was not psychotic. Insight into his illness was very poor. He actually was complaint about previous history of nausea, palpitations and sleep disturbance. Patient prior psychiatric history was remarkable with some episodes od depression which remains undetected and untreated by health care professionals. Results on Beck depression inventory of 51 scores indicate depressive disorders with severe feature-recurrent.

\subsection{Outcome and follow up}

He presented frequently mixed clinical feature of panic and heart attack followed with dyspnea, shortness of breathing, insomnia and dizziness. He had poor compliance with medical treatment including cardiologic treatment as result of depressed mood and lack of violation. The cardiac complications in terms of heart failure on such prolonged and untreated stress and depression was remarkable. He repeated some episodes of mixed panic and heart attacks and in next few days he was hospitalizated for cardiologic decompensated heart provoked by intensive psychological stress event and impact of depression in heart attack.

\section{Discussion}

Depression is an independent risk factor for morbidity and mortality, and should be taken into account by those who provide the Congestive Heart Failure treatment. Studies have found moderate up to high prevalence of depression among HF patients, which condition also presents an increased risk of mortality and cardiac crisis in HF patients [1] and may be associated with increased medical costs, hospital readmissions and decline in health status [2]. Abundant 
evidence suggests that depression is under-recognized and untreated in cardiac populations [1]. Psychosocial factors such as poor drug compliance, scarcity of social support with the low socioeconomic status were independently correlated with depressive symptoms and have also been implicated in the progression of heart failure [2]. The recognition and treatment of depression may be important component of HF care [3], as studies evidence findings showed high rates of depressive symptoms in HF patients. O'Connor and Joyn't reported that the prevalence of depression ranges from $11 \%$ to $25 \%$ for outpatient and from $35 \%$ to $70 \%$ for inpatient [3], while only $6.6 \%$ of the general population have meet the criteria for depressive disorder based on the 12-month study [4]. This suggests that depression can exacerbate prognosis and precipitate HF development.

Psychosocial factors may also contribute for example: depression is associated with poor therapeutic compliance, scarce social support and higher prevalence of cigarette smoking, and all of these were correlated with the HF worsening [3]. Prognosis and the progress of depression is influenced by poor cooperation between patient and his doctor as regards the lifestyles and medication intake of a patient [5]. Safety and efficacy of antidepressant therapy in patients with CVD are not well established. Evidence from clinical studies evaluating the impact of pharmacological and behavioral treatment of depression on morbidity and mortality of HF patients are limited [5]. Some earlier studies suggests that SSRI antidepressant therapy may improve cardiac outcomes in depressed FH patients, but further study is needed [6]. Detection and treatment of depression should be part of a comprehensive approach to heart failure patients by cardiologists and family doctors. High quality cardiac care and management of HF case should include the assessment of psychosocial factors, strengthening of the doctor-patient relationship and of family and social bonds, and, when appropriate, antidepressants and psychotherapy [7]. Nevertheless, reducing of depression symptoms could be expected to improve compliance to HF treatment regimens [1].

\subsection{Non adherence to treatment}

a) Not initiating a recommended treatment

b) Not attending or coming late to appointments

c) Not completing behavioral recommendations or homework (e.g. increase in physical activity, changes in diet, self monitoring, in vivo exposure, relaxation exercise)

d) No taking medication as prescribed (e.g. taking too many or too few pills, taking medication at incorrect times, not following special dosing instructions)

e) Terminating the treatment prematurely.

Major depression should not be recognized as normal mental reaction to illness but as a disorder that causes chronic disability otherwise treatable condition that it is [3]. The depression is more likely to increase the mortality and morbidity through a specific mechanisms such is poor medical therapeutic compliance rather than through biological mechanisms affecting cardiovascular disease risk [8]. In medical treatment literature, rates of non adherence to treatment have generally been found to be $20-40 \%$ for acute regimen, $30-60 \%$ for chronic regimens, and $50-80 \%$ for preventive regimens. Compared with non depressed patients, the odds are 3 times greater that patients with depression will have poor medical therapeutic compliance with doctors recommendations. Evidence suggesting of 
strong covariation between depression and poor medical therapeutic compliance shows that depression as risk factor for poor outcomes among HF patients should be recognized and to underwent medical advice [10]. Poor medical therapeutic compliance and lifestyle recommendations are major problems in patients with HF [9] and many studies suggests that this inappropriate behaviour is common in CHF patients and leads to increased hospitalization and even death [11]. Evidence based interventions to improve compliance in patients with HF are scarce. Therapeutic interventions that have impact on increasing of poor medical therapeutic compliance and prevent HF worsening and related readmission in order to improve the quality of life of patients with HF need to be developed and tested [9].

\subsection{Learning points}

- Depressive disorders are associated with poor adherence, and in this case report the treatment management of CHF was very challenging.

- In terms of mortality and disability, adherence with treatment has rarely been the object of specific research, especially when compared with the vast amount of studies on the effectiveness of antidepressive drugs.

- The few quantitative studies on adherence (non-randomized and randomized interventions) do not provide either reliable or consistent indications as to the efficacy of specific interventions or combinations thereof.

- We have to take more care on early detection of depression in CHF increased risk patients and to describe psychological profile of patients that are presenting with non adherence to treatment behavior in order to prevent outcomes and early death.

\section{References}

1. Brian L Mishara. Indirect suicide. Enciclopedya of death and dying. Suicide types (2002).

2. Mark W Ketterer, Knysz W, Keteyian SJ, et al. Cardiovascular Symptoms in Coronary-Artery Disease Patients Are Strongly Correlated With Emotional Distress. Psychosomatics 49 (2008): 230-234.

3. Thomas Rutledge, Veronica A Reis, Sarah E Linke, et al. Depression in Heart Failure: A Meta-Analytic Review of Prevalence, Intervention Effects, and Associations with Clinical Outcomes. J Am Coll Cardiol 48 (2006): 1527-1537.

4. Abiodun O Adewuya, Bola A Ola, Olufemi E Ajayi, et al. Mosaku: Prevalence and Correlates of Major Depressive Disorder in Nigerian Outpatients With Heart Failure. Psychosomatics 47 (2006): 479-485.

5. O'Connor CM, Joynt KE. Depression: Are we ignoring an important comorbidity in heart failure?. J Am Coll Cardiol 43 (2004): 1550-1552.

6. Kessler RC, Berglund P, Demler O, et al. The epidemiology of major depressive disorder: Results from the National Comorbidity Survey Replication (NCS-R). JAMA 18 (2003): 3095-3105.

7. Szygula Jurkiewicz B, Duszanska A, Polonski L. Is depression a problem in patients with chronic heart failure?. Pol Arch Med Wewn 118 (2008): 52-56.

8. Jiang W. Impacts of depression and emotional distress on cardiac disease. Cleve Clin J Med 75 (2008): 2025 . 
9. Cardoso G, Trancas B, Luengo A, et al. Heart failure and depression :an association with clinical importance. Rev Port Cardiol 27 (2008): 91-109.

10. David J Vinkers, Jacobijn Gussekloo, Max L Stek, et al. Does Depression Specifically Increase Cardiovasculare Mortality?. Arch Intern Med 165 (2005): 119.

11. van der Wal MH, Jaarsma T, van Veldhuisen DJ. Non-compliance in patients with heart failure; how can we manage it?. Europian Journal of Heart Failure 7 (2005): 5-17.

12. Robin DiMatteo M, Heidi S Lepper, Thomas W Croghan. Depression Is a Risk Factor for Noncompliane With Medical Treatment Meta-analysis of the Effects of Anxiety and Depression on Patient Adeherence Arch Intern Med 160 (2000): 2101-2107.

13. MacFadyena RJ, Fraser CG, Struthersb AD. Short report: Intermittent non-adherence with ACE inhibitor treatment and implications for clinical trials resultes. Heart 85 (2001): 213-214.

14. Amam Mbakwem, Francis Aina, Casmir Amadi. Expert Opinion: Depression in Patients with Heart Failure: Is Enough Being Done?. Cardiac failure review 2 (2016): 110-112.

15. Meltem Refiker Ege, Nuryil Yilmaz, Mehmet Birhan Yilmaz. Depression and heart failure. int $\mathrm{j}$ of cardiology 158 (2012): 474.

16. Nebi R Musliu, Nazmie F Ibishi, Zylfije Hundozi. Depression and heart failure: decline in quality of life and future management considerations. Cardiologia croatica 8 (2013): 3-4.

Citation: Nazmie F Ibishi, Nebi R Musliu, Shpend Haxhibeqiri, Bujar Obertinca. A 57 year Old Man with Depressive Disorder, Congestive Heart Failure and Non Adherence with Treatment. Journal of Psychiatry and Psychiatric Disorders 3 (2019): 001-005.

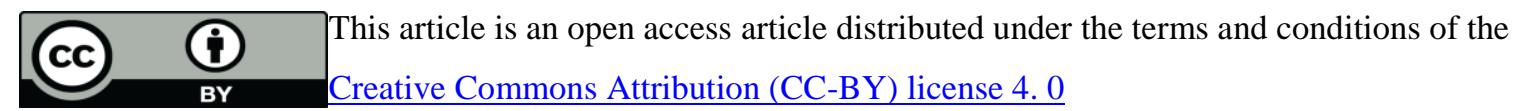

